\title{
Wind Turbines Condition Monitoring and Fault Identification using Acoustic Emission Testing Method
}

\author{
Qin Hongwu ${ }^{1}$, Kong Lingbo ${ }^{2}$, Zhang $\mathrm{Xian}^{3}$ and Fan Qinyin ${ }^{4}$ \\ ${ }^{1}$ College of Electronic and Information Engineering \\ ${ }^{2}$ College of Machinery and Vehicle Engineering \\ ${ }^{3}$ International Education College, Changchun University, Changchun, China \\ ${ }^{4}$ Graduate School of Engineering, Osaka University, Osaka, Japan \\ hongwuqin@live.cn,fqyyhoo@yahoo.co.jp
}

\begin{abstract}
Acoustic Emission (AE) technique can successfully applied for condition monitoring of low speed rotating components such as rolling bearing and gearbox of Wind Turbines. This technique is able to detect very small energy released rates from incipient defect in a very early stage. Wide range of signal processing methods can be apply for diagnosing faults and fatigues in AE spectrums and the changes in wave forms are very significant to recognize the failures. Condition monitoring and Fault identification (CMFI) of wind turbine health using automated failure detection algorithms can improve turbine reliability. AE testing is based condition monitoring system uses data already collected at the wind turbine controller. It is an effective way to monitor wind turbines for early warning of failures and performance issues. CMFI methods are classified into modelbased and signal-based methods. They can be implemented with or without the use of artificial intelligence. The object of this thesis is to design a model-based CMFI scheme for WTs, which can be used under normal operation conditions.
\end{abstract}

Keywords: AE, WT, CMFI, ARMA, condition monitoring systems, AFC

\section{Introduction}

Acoustic Emission (AE) as a technique in the sounds associated with the failure of materials was detected. Acoustic wave is a non-stationary signal which can discover elastic stress waves in a failure component, capable of online monitoring, and is very sensitive to the fault diagnosis. The Acoustic Emission (AE) technique is very adequate to detecting extremely miner energy release rates. On the basis that a significant change is indicative of a developing failure, condition monitoring systems (CMS) comprise combinations of sensors and signal processing equipment that provide continuous indications of component (and hence wind turbine) condition based on technique AE.

Condition Monitoring and Fault Detection (CMFI) is a topic that becomes increasingly important in industrial processes, due to growing demands on operational reliability, safety and product quality. The general idea is to use a scheme based on measured process data to detect a fault occurrence in a physical process, e.g. a component deterioration, to isolate the fault location in the process and to identify the time characteristics of the fault. The reliability of a wind turbine can be increased by making the construction more robust. However, since enlarging the current design already leads to severe material costs; it is certainly not desirable to make even heavier constructions. To make offshore wind turbines feasible, a lighter construction must be designed that has a higher reliability than the current generation of wind turbines. Lighter and stronger materials have to be developed to achieve this ambitious goal; a large challenge for materials scientists. Another large step forward can be made by control engineers: the design of better 
maintenance schemes for turbine components can lead to cost reduction and higher availability of the turbine.

As is well-known, spectrum of signals sum equals sum of spectra, hence effective width of summarized signal spectrum should not increase. However, its distortion is considerable. Transfer properties of the acoustic tract are described by frequency response function (FRF). Classical method of frequency distortion influence exclusion consists of FRF calculation with subsequent adjustment of received signals spectral characteristics. Plane shape objects FRF can be calculated theoretically. Let us do FRF calculations for a long road. The practical use of acoustic emission method for technical diagnosis's purposes presents some difficulties. An acoustic signal spreading in object undergoes an essential distortions caused by "object-converter" system. These distortions are so considerable that to retrieve the true shape of signal is almost impossible in practice. So the problem of searching correlations between construction's status variables and signal characteristics, and the problem of acoustic signal identification are important today.

Let's try to prove the necessity of AE signal analysis in broad band. It is well-known that material $\mathrm{AE}$ is the process of producing elastic waves, provoked by local dynamic reconstruction of its structure. It is important to give much attention to cracks formation and development as emergency situations and breakdowns at industrial facilities are commonly stipulated by formation and further development of cracks in material of the survey item. Increased efficiency and reliability of modern industrial equipment with one hand, makes it necessary to use him in conditions close to the limit, and, with another requires a fairly accurate determination of the stress-strain state in the test facility for timely identification of critical situations and prevent the destruction of. Therefore, an important challenge is the development of various non-destructive control methods for assessing the strength properties of materials in the operation of structures without destroying its integrity. The experience of recent decades has shown great potential of the method of acoustic emission, which is simple to use, economical and provides continuous monitoring of the growth of latent defects in structures during their construction and industrial use.

Most WT machines are three-blade units comprising the major components illustrated in Figure1 Driven by the wind, the blades and rotor transmit energy via the main shaft through the gearbox to the generator, the main shaft being supported by the bearings, and the gearbox being such that the generator speed is as near as possible to optimal for the generation of electricity. Alignment with the direction of the wind is controlled by a yaw system and the housing is mounted at the top of a tower.

Some defects such as leaking and corrosion can be detected by visual inspection; discoloration of component surfaces may indicate slight temperature variations or deteriorating condition, and the sound coming from the bearings can also indicate physical condition. However, many of the most typical failures like cracking and roughness on the surfaces of the blades, electric short circuits in the generator, and overheating of the gearbox all demand a more sophisticated approach to maintenance.

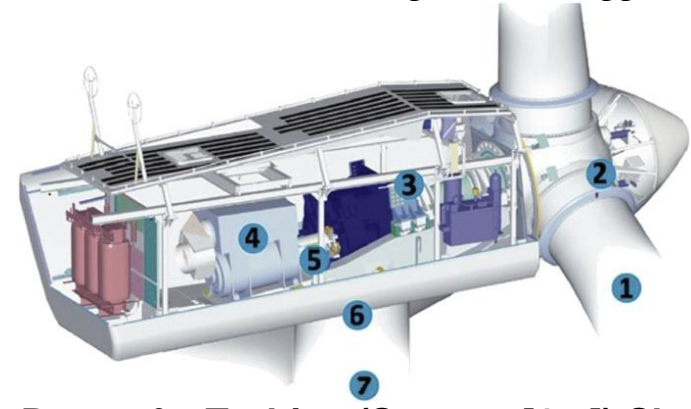

Figure 1. Main Parts of a Turbine (Source: [1-7]) Showing (1) Blades, (2) Rotor, (3) Gearbox,(4) Generator, (5) Bearings, (6) Yaw System and (7) Tower 


\section{Experimental Data}

We carried out experiments which have demonstrated that applying acoustic emission parameters based on impulses reading for quantitative acoustic emission description principles invariably results in low researches results precision. Such situation in measurements may occur in the presence of not excluded systematic inaccuracies and bear the evidence of incorrectness of such measurements due to improper choice of physical units characterizing acoustic emission properties. If to compare main acoustic emission measurement units with the International System of units, it can be seen that generally accepted acoustic units are almost never used for acoustic emission description. Upon switching to International System of units there was some regulation in values and units usage characterizing ionizing radiation and its field, and that increased measurement results reliability. Unfortunately, that did not happen in the sphere of acoustic emission. All existing normative documents starting from GOST 2763-83 and ending with rather up-to-date RD 03-131-97 and RD 03-299-99 recommend using parameters based on impulses counting.

We are bound to analyze only the part of a collective process which appears to be above the equipment sensitivity threshold. This fact gives rise to multiple speculations and scientific fantasies. Mostly we can only see the tip of an iceberg and the rest data is left behind-the-scenes, under sensitivity threshold. As a result, even genuine values of such AE signal physical quantities as pulse height and energy are frequently do not allow establishing any correlations. We need comprehensive knowledge of process physics to evaluate the whole process by available data. It is important to realize that forming of the "tip" is indissolubly related to sensibility and frequency characteristic of a converter, method and accuracy of converter positioning, AE properties of a material and wave characteristics of a survey item, as well as to loading dynamics and structural in homogeneity of the material. We also have to take into consideration that AE formation process is comprised by several simultaneous processes part of which are auxiliary and depend on environmental conditions.

Currently, a number of methods of modeling of Gaussian processes, among which the most frequently used ARMA-model because of the simplicity of its representation, as this model include a small number of parameters. The essence of this method is applied to the original Gaussian signal (noise) some shaping filters.)

The general form of a discrete stochastic process $\mathrm{x}(\psi)$ can be described by a generalized operator equation autoregressive - moving average (ARMA):

$$
X(t)=A_{p}^{-1}(B) C_{q}(B) E(t)
$$

where $A_{p}(B)$ - the operator of order autoregression, $C_{q}(B)$ - moving average operator of order q, E ( $\mathrm{t}$ ) - a sequence of independent random variables with zero mean and unit variance with normal or uniform distribution.

Notable studies ARMA-model limited to the qualitative side of the issue and do not deal with virtually influence the statistical characteristics of the current at the input of the film-forming white noise on the statistical properties of implementations and evaluation of model errors.

In Figure 2 shows the graphs of the basic statistical parameters (mathematical expectation $M$ dispersion $D_{2}$, autoregression $\rho(m)$ ) of the random process $E(t)$ with a uniform distribution on the interval from -0.5 to 0.5 and a length $\mathrm{N}=400$. 

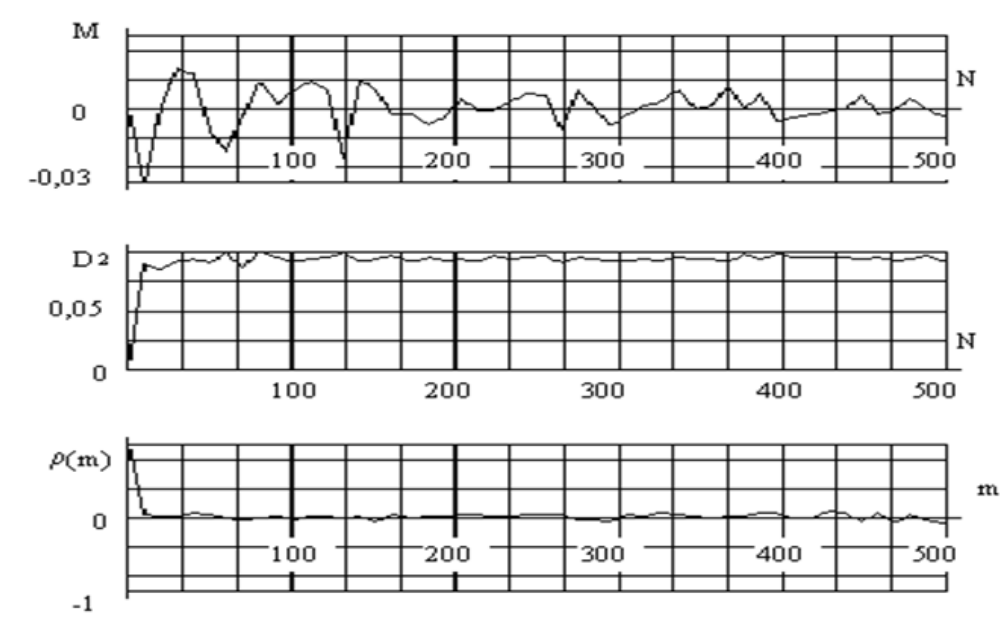

\section{Figure 2. Statistical Characteristics of a Random Process with a Uniform Distribution}
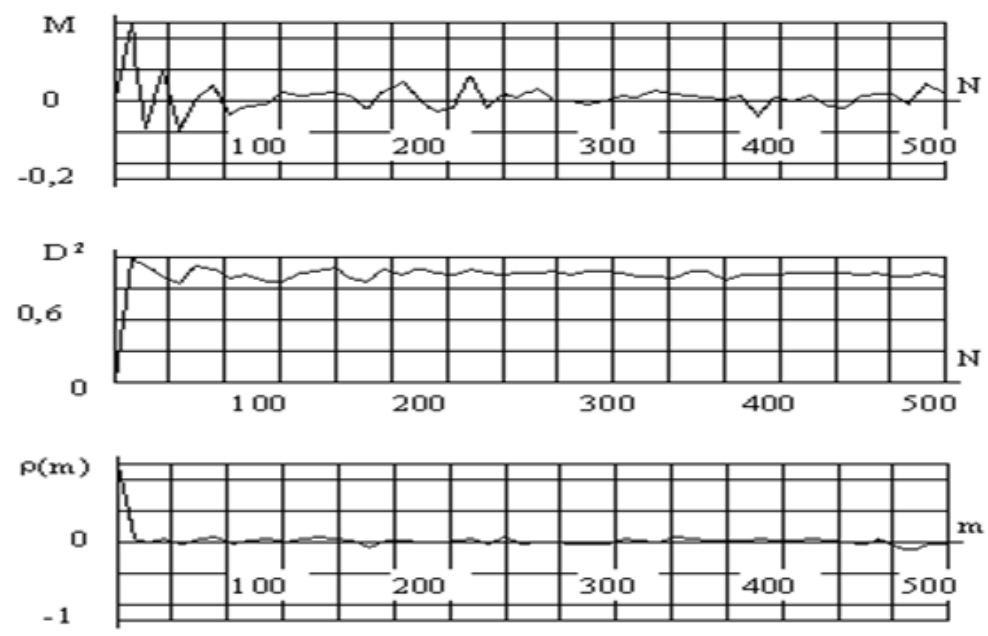

\section{Figure 3. Statistical Characteristics of a Random Process with a Normal Distribution}

Similar results (analogous results) for the implementation of white noise with a normal distribution $\mathrm{N}\left[0, \sigma^{2}\right]$ shown in Figure 3 From these results it follows that the first order statistical moments are stabilized at $\mathrm{N} \geq 100$ for a process with a uniform distribution and with $\mathrm{N} \geq 50$ for a normal distribution process. It should be emphasized that the results obtained by averaging over ten realizations of the process.

Depending on the desired statistical characteristics of stationary measuring signal can be described as ARMA-, AR-or MA-process with a finite number of defining parameters. Most often used in technical applications AP-model random processes not over the second order.

For some applications an important parameter of the measurement signal is the spectral characteristic. The spectral density of the ARMA process defined by the expression:

$$
S_{A R M A}(\omega)=\sigma_{t}^{2} \frac{\left(1-\sum_{i=1}^{q} c_{i} \cos i \omega\right)^{2}+\left(\sum_{i=1}^{q} c_{i} \sin i \omega\right)^{2}}{\left(1-\sum_{k=1}^{p} d_{k} \cos k \omega\right)^{2}+\left(\sum_{k=1}^{p} d_{k} \sin k \omega\right)^{2}}
$$


where $c_{i}-i$-th coefficient of the moving average, $d_{i}-i$-th coefficient of autoregression, $\mathrm{q}$ and $\mathrm{p}$ - orders shaping filters and autoregressive moving average, respectively.

From the above formula it follows that for AR processes shaping filter amplitudefrequency characteristic (AFC) at $\sigma_{2}=1$ is described by:

$$
S(\omega)=\left[\left(1-\sum_{K=1}^{P} d_{k} \cos k \omega\right)^{2}+\left(\sum_{k=1}^{p} d_{k} \sin k \omega\right)^{2}\right]^{-1}
$$

In Figure 4 shows the form of changes in AFC of AR (2) process, depending on the parameter $\mathrm{a}_{1}$, at two fixed values of the parameter $\mathrm{a}_{2}$.
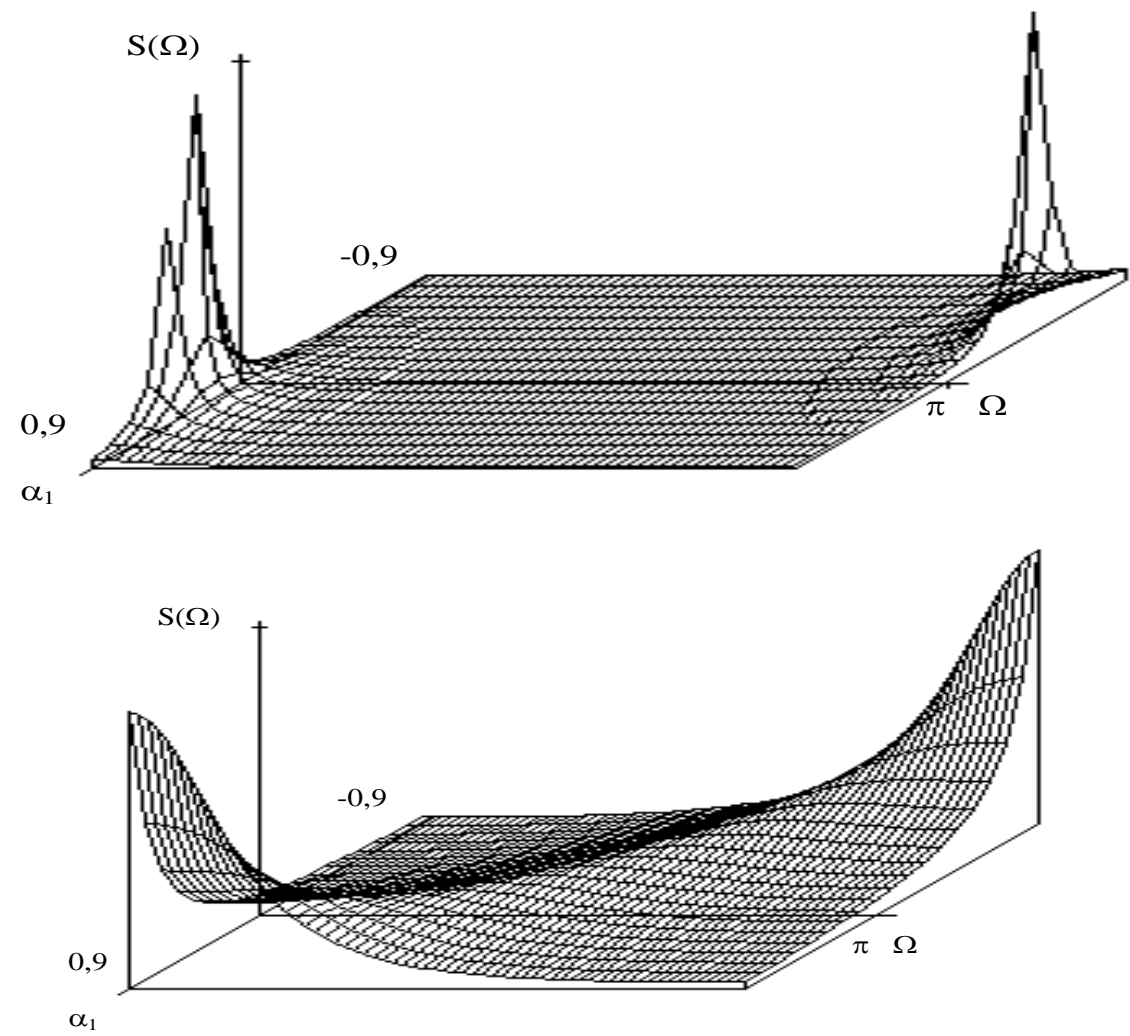

Figure 4. Characteristics AFC AR (2) Process for one Fixed Parameter

To identify opportunities for representation of signals in the form of ARMA-models necessary to determine their inherent uncertainty. Since the most commonly used model of white noise, which is formed on the basis of ARMA-process are not smooth spectral functions, characterized by significant irregular emissions. Such distortion of the spectrum inherited ARMA process and influence the shape of its spectrum. Obviously, for the ARMA processes are characterized by errors arising due to the finite size of the sample, unsteadiness original finite-dimensional realization of white noise, and also due to computational errors. 


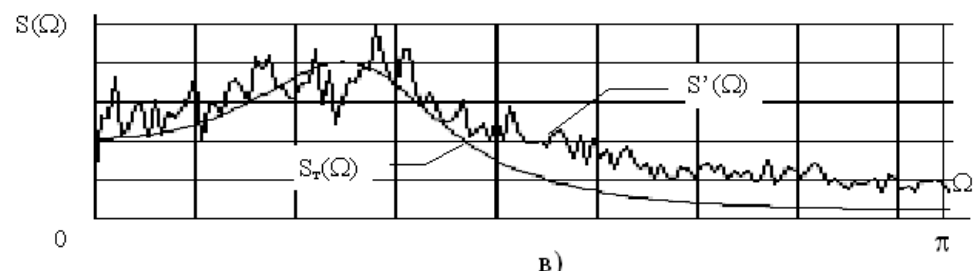

B)

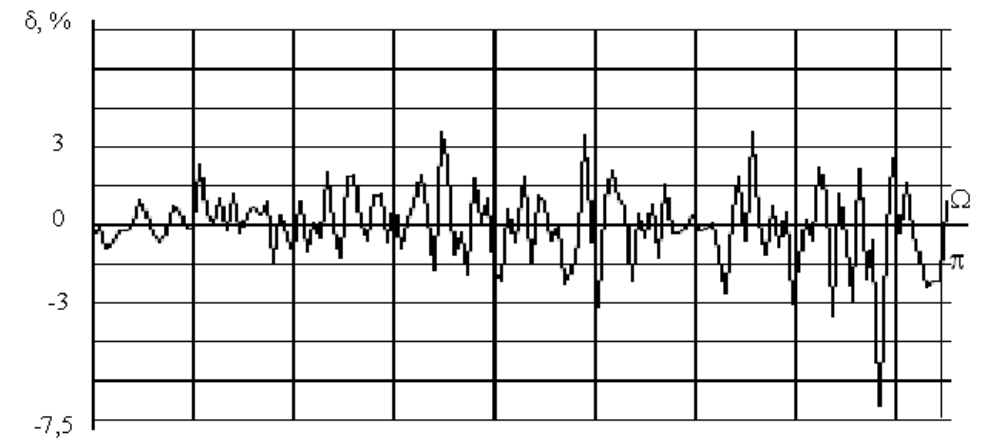

Figure 2.4 Example of Theoretical and Practical AFC AR (2) Process and the Appropriate Representation of the Relative Error

In Fig.2.4 shows an example of the theoretical ST $(\Omega)$ and real-S' $(\Omega)$ spectra for AR (2) process, as well as the corresponding relative error $\delta(\Omega)$, calculated from the spectral region. It should be noted that the actual spectrum was determined by averaging over 10 realizations of the AR (2) process.

From these studies suggest that the use of known computational models of random processes with no averaging simulation results lead to significant errors in the signal representation in the spectral domain even for large sample sizes. Thus, the models considered random processes have a marked decrease in errors in sample size $\mathrm{N}>128$ and assessments over the ensemble. For AR processes with sample size $\mathrm{N}=128$ the number of averages should be at least 20-25, and for $\mathrm{N}=512$ - no longer than 5-10.

\section{Conclusion}

CMFI methods are classified into model-based and signal-based methods. They can be implemented with or without the use of artificial intelligence. The object of this thesis is to design a model-based CMFI scheme for WTs, which can be used under normal operation conditions. It can be concluded, that model-based CMFI for wind turbine systems is an open research field. A feasibility study of model-based CMFI methods might yield interesting new methods, which can be used to monitor the condition of the components of a WT.

A study of prior CMFI research showed, that model-based CMFI has not yet found its way to application in the field of wind turbine engineering. A short description was given of existing CMFI schemes for monitoring the condition of various turbine components: rotor blades, drive train, gear box and generator. Only signal-based methods were described in literature, as well as methods based on artificial intelligence. Moreover, most of these methods use additional sensors and actuators, or can only be applied under special measurement conditions.

Conclusions made are fortified by performed theoretical and experimental analysis of AE signal spectrum properties. Taking into consideration all above mentioned I think that: 
1. Because the spectrum of the input signal is strongly dependent on the propagation conditions in the test object, before measuring $\mathrm{CX} \mathrm{AE}$ signals is necessary to evaluate AFC of the system "sample - receiving transducer."

2. The spectral characteristics of AE signals should use broadband transducers with the rise in the AFC side of the treble to compensate for AFC of the sample, and partly of the signal spectrum, which will reduce the measurement error of the absolute values.

3. If conditions allow the problem, it is preferable to restrict relative measurements of the spectral characteristics of the signals $\mathrm{AE}$, which will reduce the measurement error.

\section{Acknowledgement}

The work was supported by Jilin Provincial Science and Technology Department (No. 20140414025GH). The research activities have been funded by Changchun Science and Technology (No. 13GH15). The research activities also have been funded by Jilin Provincial Education Department (No. 2014290).

\section{References}

[1] M. R. Gorman, "Plate wave acoustic emission", JASA, vol. 90, no. 1, (1991), pp. 358-364.

[2] H. V. Ravindra, "Some aspects of acoustic emission signal processing", Journal of Materials Processing Technology, vol. 109, (2001), pp. 242-247.

[3] A. Terchi, Y. H. J. Au, "Acoustic emission signal processing”, Measurement and Control, vol. 4, no. 8, (2001), pp. 240-244

[4] B. Tomasz and Z. Dariusz, "Application of wavelet analysis to acoustic emission pulses generated by partial discharges", IEEE Transactions on dielectrics and electrical insulation, vol. 11, no. 3, (2004), pp. 433-449.

[5] E. P. Scrrano and M. A. Fabio, "Application of the wavelet transform to acoustic emission signals processing”, IEEE Transaction on signal processing, vol. 44, no. 5, (1996), pp. 1270-1275.

[6] K. Ono, Q. X. Huang, "Pattern recognition analysis of acoustic emission signals", Progress in Acoustic Emission VII, The Japanese Society for NDI, (1994), pp. 69-78.

[7] R. M. Belchamber, "Evaluation of pattern recognition analysis of emission from stressed polymers and composites", J. Acoustic Emission, vol. 4, no. 4, (1985), pp. 71-84.

[8] C. R. L. Murthy, "Application of pattern recognition concepts to acoustic emission signals analysis", J. Acoustic Emission, vol. 6, no. 1, (1987), pp. 19-28.

[9] Y. Ding, R. L. Reuben and J. A. Steel, "A new waveform method for analysis for estimating AE wave arrival times using wavelet decomposition”, NDT\&E International, vol. 37, no. 4, (2004), pp. 279-290.

[10] M. Surgeon, C. Buelens and M. Wevers, "Waveform based analysis techniques for the reliable acoustic emission testing of composite structures", Journal of Acoustic Emission, vol. 18-19, (2001), pp. 34-40.

[11] Q. F. Lu, "Model of stator inter-turn short circuit fault in doubly-fed induction generators for wind turbine", Proceedings of IEEE PESC, (2004); Aachen; Germany.

[12] L. M. Popa, "Condition monitoring of wind generators", Proceedings of IEEE IAS, (2003); Salt Lake City, USA.

[13] S. G. M. Krämer, "Fiber optic sensor network for lightning impact localization and classification in wind turbines", Proceedings of IEEE ICMFIIS, (2006); Heidelberg; Germany.

[14] M. E. H. Benbouzid, "A review of induction motors signature analysis as a medium for faults detection", IEEE Trans. Industrial Electronics, vol. 47, no. 5, (2000), pp. 984-993.

[15] D. Casadei, "Experimental fault characterization of doubly fed induction machines for wind power generation", Proceedings of IEEE SPEEDAM, (2006); Taormina, Italy. 
International Journal of Control and Automation Vol. 8, No.4 (2015) 